\title{
Comparison of intraocular pressure measurements with the portable PTI00 noncontact tonometer and Goldmann applanation tonometry
}

This article was published in the following Dove Press journal:

Clinical Ophthalmology

2 May 2009

Number of times this article has been viewed

\section{Sarwat Salim \\ Daniel J Linn \\ James R Echols II \\ Peter A Netland}

Hamilton Eye Institute, University of Tennessee Health Science Center, Memphis, TN, USA
Correspondence: Peter A Netland Siegal Professor, Hamilton Eye Institute, University of Tennessee Health Science Center, 930 Madison Avenue, Suite 100, Memphis, TN 38I63, USA

$\mathrm{Tel}+\mathrm{I} 9014485492$

Fax + I 90I 4489350

Email pnetland@gmail.com
Purpose: Noncontact tonometers are useful when regulations preclude use of contact tonometers by medical students and other nonophthalmologists. Our study compared the measurements by the portable, noncontact tonometer (PT100) with Goldmann applanation tonometry (GAT).

Methods: This was a prospective study of 98 eyes from 98 patients. Intraocular pressure (IOP) was measured by GAT and the PT100 (Reichert, Buffalo, NY).

Results: Mean IOP measurements showed no significant differences in measurements performed by the two tonometers $(\mathrm{P}=0.64)$. Measurements by the two tonometers were in agreement by $\leq 3 \mathrm{mmHg}$ in $92.8 \%$ of eyes. Linear regression analysis of PT100 vs GAT measurements revealed a slope of 0.98 with $\mathrm{r}^{2}=0.58$. Bland-Altman analysis showed a mean difference of measurements by GAT and PT100 of $-0.3 \mathrm{mmHg}$ with two standard deviation = $7.1 \mathrm{mmHg}$.

Conclusion: The portable noncontact PT100 tonometer provides IOP measurements comparable to GAT within the normal range of IOP.

Keywords: applanation tonometer, intraocular pressure, noncontact tonometry

\section{Introduction}

The Goldmann applanation tonometer (GAT) has long been considered the most accurate and dependable instrument for determining intraocular pressure (IOP). ${ }^{1}$ Noncontact tonometers (NCTs) became available for clinical use over 30 years ago. ${ }^{2}$ IOP is measured based on the force required to applanate the cornea by a puff of air. Highly trained personnel are not needed to obtain measurements, and NCTs require no anesthetic or staining, which reduces the possibility of damaging the corneal surface and cross contamination. In one study, noncontact tonometry was found to be comparable to GAT in eyes after refractive surgery. ${ }^{3}$

Since their introduction, NCTs have undergone technological improvements, simplifying their use and enhancing overall accuracy. The portable NCT, Reichert PT100 (Buffalo, NY), has been used in community screenings supported by the Friends of the Congressional Glaucoma Caucus and the Student Sight Savers Program in the United States. Although various studies have demonstrated the accuracy and precision of noncontact or "air puff" tonometers in measuring IOP and their correlation with GAT, ${ }^{4-6}$ only one recent article has reported comparable performance of PT100 and GAT. ${ }^{7}$ The purpose of this study was to further assess the agreement in IOP measurements between GAT and NCT as measured by the PT100. 


\section{Methods}

This was a prospective comparative consecutive case series. The study population was recruited from Comprehensive Service of UT Department of Ophthalmology in Memphis, Tennessee. Patients with corneal diseases and previous corneal surgery were excluded from the study. These patients did not have glaucoma based on medical history or previous exams. Institutional Review Board committee approval was obtained.

Patients signed a written consent to have their IOP checked via both the PT100 and GAT techniques, and one eye (right eye) was arbitrarily chosen for analysis. Patients were recruited from both morning and afternoon clinics and IOP measurements were obtained at different time intervals. A medical student operated the PT100, while the Goldmann tonometers were operated by trained residents and fellows in the clinics. All IOP readings were taken in the sitting position over fifteen minutes in a masked manner. NCT was performed before the GAT to avoid the known mild reduction of IOP by anterior chamber compression with GAT. ${ }^{8}$

In order to minimize the effect of cardiac pulse on IOP when using the PT100, an average of three readings was recorded, as recommended by the manufacturer. ${ }^{6}$ Any measurement with low confidence interval, as marked with an asterisk, or any out of class reading (varying by $4 \mathrm{mmHg}$ compared to other measurements), marked in brackets, was repeated. After instillation of topical proparacaine drops, GAT was performed according to standard protocol, using a Haag-Streit slit lamp, which was calibrated according to the instructions and schedule provided by the manufacturer. Only the first GAT measurement on each eye was used for the GAT data since previous literature has reported that serial readings using the GAT result in a statistically significant decrease in final IOP measurements. ${ }^{8}$

An unpaired T-test was performed to compare the mean IOP measurements obtained with two methods. Linear regression analysis of the data from the two instruments was performed. The two techniques were compared using the Bland-Altman Test for Correspondence. Statistical analysis was performed using SPSS (SPSS Inc, Chicago, IL) and Primer of Biostatistics software. P values $<0.05$ were considered statistically significant.

\section{Results}

A total of 98 eyes in 98 patients were studied. The mean age was $62.6 \pm 13.8$ years. The study population consisted of $61 \%$ men and $39 \%$ women; $56 \%$ African-American and $44 \%$ Caucasian. The mean \pm SD intraocular pressure measurements were $15.98 \pm 5.48 \mathrm{mmHg}$ and $15.65 \pm$ $4.26 \mathrm{mmHg}$ for the PT100 and GAT, respectively $(\mathrm{P}=0.64)$. The median IOP was $15.0 \mathrm{mmHg}$ for the GAT and 14.3 for the PT100 measurements. The range of measurements by GAT was from 4 to $29 \mathrm{mmHg}$ and by PT100 was 7 to $33 \mathrm{mmHg}$.

A linear regression analysis of PT100 and GAT measurements is shown in Figure 1. Linear regression analysis of PT100 vs GAT measurements revealed a slope of 0.98 with $\mathrm{r}^{2}$ of 0.58 , indicating comparable performance between the two instruments. The proportion of eyes with GAT measurement of IOP $\geq 21 \mathrm{mmHg}$ detected by PT 100 was $8 / 8$ $(100 \%)$ and the proportion of eyes with GAT measurement of IOP $<21 \mathrm{mmHg}$ detected by PT100 was 79/90 (87\%). The frequency of measurements by the two tonometers that were in agreement by $\leq 3 \mathrm{mmHg}$ was $92.8 \%$.

Results of the Bland-Altman plot are shown in Figure 2. The mean difference between the measurements in eyes by the different techniques was $-0.3 \mathrm{mmHg}$, with two $\mathrm{SD}=7.1 \mathrm{mmHg}$. The difference between measurements appeared to increase with increasing IOP, suggesting an increase in variation as the magnitude of the measurements increased.

\section{Discussion}

The hand-held Reichert PT100 allows portable noncontact tonometry. The role of tonometry in glaucoma screening has been controversial and appears to be of limited diagnostic value as a solitary test. ${ }^{9}$ Nevertheless, it is commonly performed in glaucoma screenings in conjunction with other diagnostic modalities. ${ }^{10,11}$ Screenings may utilize nonmedical and unlicensed personnel. Since NCT does not require topical anesthetic or staining drops and the readings are largely operator independent, it allows screenings to be implemented without the direct supervision of medical doctors, allowing screening staff relative autonomy in operation. ${ }^{12}$ In this study, we found that the portable NCT tonometer provided measurements of IOP that were comparable to GAT in normal patients.

Previous comparative studies of IOP measurements recorded with NCT and GAT have shown clinical agreement between the two devices, ${ }^{4-6}$ with a tendency towards lower reliability with NCT in the higher pressures ranges. ${ }^{6,13}$ PT100 utilizes the same basic operating principles as the newer generation desktop models, such as the AT550 (Depew, NY, USA). In contrast to AT550, PT100 is battery operated and weighs only $1.3 \mathrm{~kg}$. AT550 measurements have been found equivalent to GAT in both normal ${ }^{14}$ and glaucomatous subjects, ${ }^{15}$ and comparable IOP measurements have 


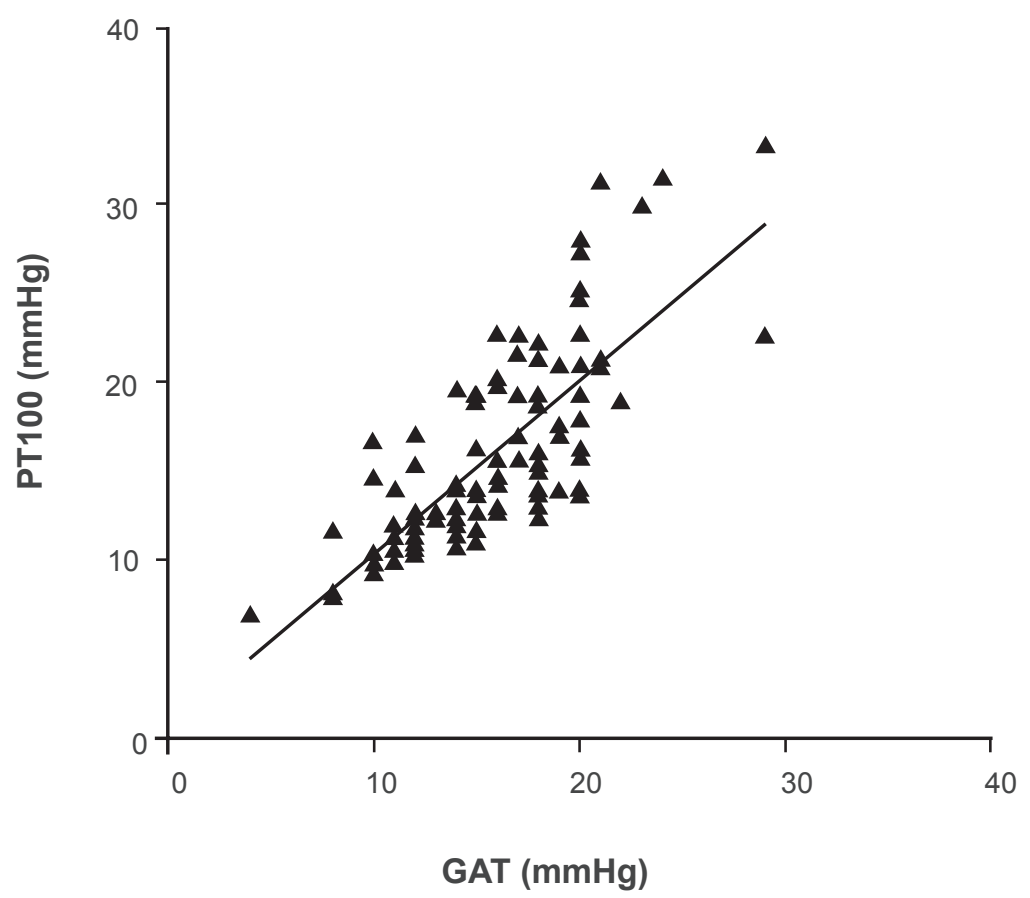

Figure I Linear regression analysis of PTI00 noncontact tonometer and GAT measurements of intraocular pressure. The slope was 0.98 with a correlation coefficient of $0.58\left(r^{2}\right)$.

Abbreviation: GAT, Goldmann applanation tonometry.

been found with portable NCTs and previous generations of NCTs. ${ }^{712}$

In our study, PT100 and GAT demonstrated a close level of agreement when compared within the normal range of IOP levels, as shown by the correlation of measurements in linear regression analysis and Bland-Altman analysis for correspondence. Bland-Altman analysis suggested increased variation with increased magnitude of the measurements. Differences in the performance of GAT and NCT have been reported with extreme range of IOP values. ${ }^{12}$ In the small

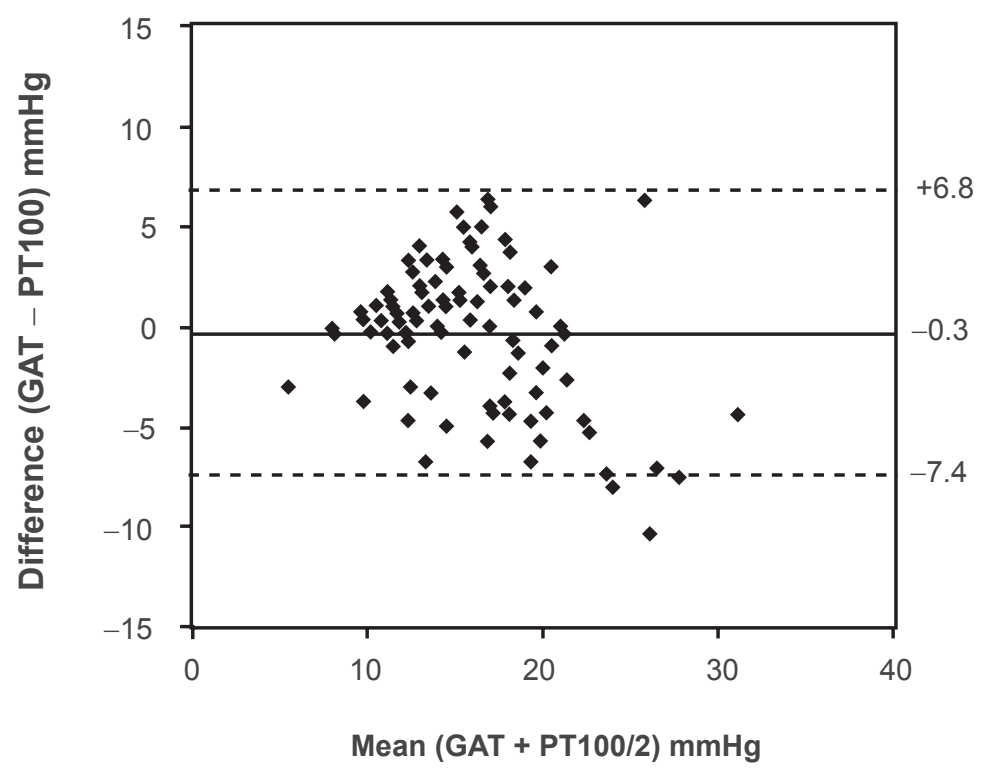

Figure 2 Bland-Altman test for correspondence of PTI 00 noncontact tonometer and GAT measurements of intraocular pressure. The mean difference of IOP was - 0.3 mmHg, with two standard deviations $=7.1 \mathrm{mmHg}$.

Abbreviations: GAT, Goldmann applanation tonometry; IOP, intraocular pressure. 
subset of eyes, the PT100 identified the majority of eyes with IOP $\geq 21 \mathrm{mmHg}$; however, further evaluation with a large sample size is needed to determine the accuracy of the device with increased IOP, especially in screening settings where candidates may present with different levels of IOP.

Potential limitations of NCT include the need for proper fixation, especially with PT100 since it does not have a headrest. Although internal indicators within the device direct centration and improved alignment, some degree of practice is required to obtain accurate measurements. The corneal surface should be regular and smooth for accurate measurements. ${ }^{16}$ One study has advised about the remote possibility of infection with NCT due to microaerosol formation. ${ }^{17}$ Another potential drawback is that all tonometers, including both GAT and NCT, are affected by corneal properties, such as corneal thickness, curvature, rigidity, and hydration. ${ }^{18}$

A limitation of this study was the relatively small numbers of eyes for analysis of measurement of increased IOP, and the possible effects of central corneal thickness (CCT) on IOP measurements. Although this study was not intended to evaluate the influence of CCT on IOP measurements, NCTs have been shown to be affected more by CCT than GAT. ${ }^{19,20}$ Murase and colleagues have reported that PT100 is more affected by variations in CCT than GAT. ${ }^{7}$

\section{Conclusion}

The PT100 noncontact tonometer provides an accurate measurement of IOP when compared with GAT within the normal range of IOP. Further studies are needed to explore these relationships in eyes with raised pressure. The PT100 has the advantage of portability, suggesting a possible use by nonmedical personnel for nonoffice-based IOP measurement.

\section{Disclosure}

Supported in part by the Friends of The Congressional Glaucoma Caucus Foundation and the Hyde Foundation. Presented in part at the American Academy of Ophthalmology Annual Meeting, Atlanta, Georgia, November, 2008. The authors have no financial interest in this work.

Clinical Ophthalmology

\section{Publish your work in this journal}

Clinical Ophthalmology is an international, peer-reviewed journal covering all subspecialties within ophthalmology. Key topics include: Optometry; Visual science; Pharmacology and drug therapy in eye diseases; Basic Sciences; Primary and Secondary eye care; Patient Safety and Quality of Care Improvements. This journal is indexed on Submit your manuscript here: http://www.dovepress.com/clinical-ophthalmology-journal

\section{References}

1. Chihara E. Assessment of true intraocular pressure: the gap between theory and practical data. Surv Ophthalmol. 2008;53:203-218.

2. Forbes M, Pico G Jr, Grolman B. A noncontact applanation tonometer. Description and clinical evaluation. Arch Ophthalmol. 1974;91:134-140.

3. Abbasoglu OE, Bowman RW, Cavanagh HD, McCulley JP. Reliability of intraocular pressure measurements after myopic excimer photorefractive keratectomy. Ophthalmology. 1998;105:2193-2196.

4. Vernon SA. Non-contact tonometry in the postoperative eye. $\mathrm{Br} J$ Ophthalmol. 1989;73:247-249.

5. Hansen MK. Clinicial comparison of the XPERT non-contact tonometer and the conventional Goldmann applanation tonometer. Acta Ophthalmol Scand. 1995;73:176-180.

6. Myers KJ, Lalle P, Litwak A, et al. XPERT NCT - a clinical evaluation. J Am Optom Assoc. 1990;61:863-869.

7. Murase H, Sawada A, Mochizuki K, et al. Effects of corneal thickness on intraocular pressure measured with three different tonometers. Jpn J Ophthalmol. 2009;53:1-6.

8. Kakau, CET, Wilke K. On repeated tonometry. Acta Ophthalmol Scand. 1971;49:611-614

9. Sponsel WE. Tonometry in question: can visual screening tests play a more decisive role in glaucoma diagnosis and management? Surv Ophthalmol. 1989;33:291-300.

10. Mundorf TK, Zimmerman TJ, Nardin GF, Kendall KS. Automated perimetry, tonometry, and questionnaire in glaucoma screening. Am J Ophthalmol. 1989;108:505-508.

11. Tuck MW, Crick RP. The cost-effectiveness of various modes of screening for primary open angle glaucoma. Ophthalmic Epidemiol. 1997;4:3-17.

12. Moreno-Montanes J, Gomez-Demmel E, Lajara-Blesa J, AlisedaPerez D. Comparative study of three non-contact tonometers and the Goldmann tonometer. Ophthalmologica. 1994;208:115-118.

13. Popovich KS, Shields MB. A comparison of intraocular pressure measurements with the XPERT noncontact tonometer and Goldmann applanation tonometry. J Glaucoma. 1997;6:44-46.

14. Jorge J, Diaz-Rey JA, Gonzalez-Meijome JM, et al. Clinical performance of the Reichert AT550: a new non-contact tonometer. Ophthal Physiol Opt. 2002;22:560-564.

15. Jorge J, Gonzalez-Meijome JM, Diaz-Rey JA, et al. Clinical performance of non-contact tonometry by Reichert AT550 in glaucomatous patients. Ophthal Physiol Opt. 2003;23:503-506.

16. Shields MB. The non-contact tonometer. Its value and limitations. Surv Ophthalmol. 1980;24:211-219.

17. Britt JM, Clifton BC, Barnebey HS, Mills RP. Microaerosol formation in noncontact “air-puff” tonometry. Arch Ophthalmol. 1991;109: 225-228.

18. Brandt JD. Corneal thickness in glaucoma screening, diagnosis, and management. Curr Opin Ophthalmol. 2004;15:85-89.

19. Ko YC, Liy CJ, Hsu WM. Varying effects of corneal thickness on intraocular pressure measurements with different tonometers. Eye. 2005;19:327-332.

20. Tonnu PA, Ho T, Newson T, et al. The influence of central corneal thickness and age on intraocular pressure measured by pneumotonometry, non-contact tonometry, the Tono-Pen XL, and Goldmann applanation tonometry. Br J Ophthalmol. 2005;89:851-854.

\section{Dovepress}

PubMed Central and CAS, and is the official journal of The Society of Clinical Ophthalmology (SCO). The manuscript management system is completely online and includes a very quick and fair peer-review system, which is all easy to use. Visit http://www.dovepress.com/ testimonials.php to read real quotes from published authors. 